DOI: 10.21554/hrr.091902

\title{
GRAMMATICAL DEVELOPMENT AND THE USE OF GRAMMATICALLY COMPLEX SENTENCES IN CHILDREN WHO STUTTER AND CHILDREN WHO DO NOT STUTTER
}

\section{Mirza Sitarevića \\ Leila Begić ${ }^{1, \mathbf{b}}$ \\ Zamir Mrkonjićb}

Original scientific paper

aPublic institution, Primary school „Duboki potok“, Srebrenik, Bosnia and Herzegovina

${ }^{b}$ Faculty of Education and Rehabilitation, University of Tuzla, Bosnia and Herzegovina

Received: 2019/6/14

Accepted: 2019/8/25

\begin{abstract}
The main aim of the research was to determine the development of grammar and the use of grammatically complex sentences in stuttering and non-stuttering children, and to determine whether there are differences in the above abilities between these two groups of respondents. The sample of respondents consisted of a total of 64 children aged 56-83 months. Respondents are divided into two groups. The experimental group consisted of 32 stuttering children, of whom 19 were male and 13 female. The control group consisted of 32 children who did not stutter, and who compared with age and gender, were equal with the respondents of the experimental group. The research was conducted in preschools and elementary schools in the area of the Tuzla and Una-Sana Cantons in Bosnia and Herzegovina. The results of the study showed that stuttering children show statistically lower abilities during repetition of sentences, as well as recognition, understanding and use of common morphological forms. However, it is important to point out that children who stutter, regardless of significantly lower results than their fluent speaking peers, have shown above-average grammatical abilities. Also, the results showed that both children who stutter and children who do not stutter in their spontaneous speech use complex sentences.
\end{abstract}

Key words: language, stuttering, syntax, morphology, complex sentence

\section{INTRODUCTION}

Stuttering is a disorder that is easily recognized as it affects the fluency of speech (Khan, 2015; Duranović, Begić, Jovanović-Simić, \& Rahmanović, 2018). It begins during childhood and, in some cases, lasts throughout life (Duranović et al., 2018). Stuttering hinders fluent speech and has the effect of influencing learning processes (Khan, 2015). The latest Diagnostic and Sta- tistical Handbook for Mental Disorders DSM-V (2013, according to Duranović et al., 2018) defines stuttering as a communication disorder that characterizes a break in normal speech fluency, where speech speed is inconsistent with what is expected in view of age. Frequent repetitions of voices, syllables and single-word words, prolongation of voices, insertion, pause within the word, hearing or silent blockade, tension and circulation, or word substitution are being reported.

\footnotetext{
Correspondence to:

${ }^{1}$ Leila Begić, PhD, Faculty of Education and Rehabilitation, University of Tuzla, Bosnia and Herzegovina

Univerzitetska 1, 75000 Tuzla, Bosnia and Herzegovina

Phone:+387 61502350

E-mail: leila.begic@untz.ba
} 
All of this is already noticeable during early development and affects school achievement and social communication.

Grammar defines language as a system of means of expression. It is common that morphology and syntax are called grammar (Crystal, 2001, 2003, 2003, McArthur, 1992, according to Newcomer \& Hammill, 2008). Morphology deals with the internal organization of words and the words consist of small units called morphs, which at the same time represent the least grammatical units (Owens, 2005). A part of the grammar that studies the syntactic structure of the language, that is, the totality of the functions of syntactic units, and their interrelationships and connections is called the syntax. The syntax is on the one hand bounded by the word and on the other hand by the bound text, and the central place in the syntax is taken by the sentence which is the basic syntactic unit (Jahić, Halilović, \& Palić, 2000). Ratner (1995) points out that in clinical or research follow-up of stuttering children, distortions of articulation of voices are most often observed, a disorder in the development of syntax and / or morphology in the language and difficulty of naming. Although it is believed that there is a correlation between stuttering and several linguistic variables, clear causal connections have not been established so far and there is no consensus on their precise role or contribution as risk factors for the occurrence of stuttering and its persistence, or their impact on natural recovery. This and several other aspects of language association and stuttering remain the subject of scientific debate and controversy (Nippold, 2004). These dilemmas have attracted very rich and diverse research activities, whose results should greatly improve understanding of stuttering and treatment planning.

Namely, numerous studies have examined whether linguistic abilities are associated with the onset and duration of stuttering. It is still not fully clear whether all those who stutter have problems in processing the language components. Although stuttering is a speech disorder, attention should be given to speaking within the context of the language. In this connection, it is necessary to conduct language skills tests in the persons who stutter, or the context within which the stuttering takes place (Kutnjak, Mance, \& LekoKrhen, 2016). Another reason for the need for additional research on the above issues is that stuttering typically begins in the age between the second and the fifth year, which is also the period of fast enrichment of vocabularies and the adoption of various morphological and syntactic structures
(Ntourou, Conture, \& Lipsey, 2011, according to Kutnjak et al., 2016). Stimulating predictability of stuttering in the language domain revealed that stuttering varied in several grammatical factors. Most stutterers stutter more often on consonants, on initial phonemes in words, on words at the beginning of a sentence, on contextual speech, on nouns, verbs, adjectives and adverbs, on longer words and accentuated syllables. Stuttering proved to be very dependent on the language that the stuttering person uses (Braun et al 1997, according to Salihović, 2005). The results of numerous studies have confirmed that stuttering children have poorer linguistic abilities than non-stuttering children (Anderson \& Conture, 2000; Pellowski \& Conture, 2005). Bajaj, Hodson and Schommer-Aiken (2004) examined grammatical awareness in pre-school children and children of first and second classes who stutter and children who do not stutter. The results showed that stuttering children showed statistically significantly lower scores on grammar tasks than the control group. Tetnowski (1998, according to Finneran, Leonard, \& Miller, 2009) assumes that speech disorders, including those considered to be normal non-fluency (e.g., repeating the phrase) and those usually associated with stuttering (e.g., repetition of parts of a word,) can be related to speech planning. Namely, factors that increase the requirements of language formulations, such as the longer length of the statement, the syntactic complexity and / or the complexity of the task, contribute to the higher incidence of speech disfluencies in non-stuttering children. On the other hand, the results of some studies have shown that there is no difference in linguistic abilities in children who stutter compared to their non-stuttering peers. Watkins, Yairi and Ambrose (1999) examined the lexical, morphological and syntactic characteristics of speech in 62 children at the age of 2 to 5 years who recovered from stuttering and 22 children with stuttering. The results showed a similarity in linguistic abilities between these two groups. Both groups showed normal expressive language abilities. Wingate (2001, according to Watkin \& Johnson, 2004) argues that most of the evidence suggests that stuttering children have language difficulties, and concluded that studies that have not found language lag in the stuttering children are methodologically wrong. Unlike these attitudes, there seems to be more and more evidence suggesting that language skills of stammering youngsters do not differ from their average expectations (Watkin \& Johnson, 2004). 
Thus, at this point there is no consensus on language difficulties as a risk factor in stuttering children, and it is still not possible to say whether the level of language development / disturbance is sufficient for early predicting the incidence and persistence of stuttering or eventual recovery. In accordance with the presented content and previous research results that have not yet been harmonized in relation to the above mentioned issue, and most of the research has been conducted in English, the aim of this research is to examine the development of grammar and the ability to use grammatically complex sentences of the Bosnian language in stuttering children and non-stuttering children.

\section{METHODS}

\section{Sample respondents}

In this study, a total of 64 children, aged 56-83 months (from 4 years and 8 months to 6 years and 11 months) were examined. Respondents are divided into two groups. The first group consisted of 32 stuttering children, 19 of whom were male and 13 female respondents (experimental group). On the basis of the experimental group, a control group was also created that included the same number of non-stuttering children that, by age and gender, were equated with an experimental group. The research was conducted in preschools and elementary schools in the area of Tuzla and Una-Sana Canton in Bosnia and Herzegovina. The test was carried out individually with each respondent according to the test instructions.

\section{Sample variables}

The variables used in this study can be divided into two groups:

1. Anamnestic variables (Stuttering children and nonstuttering children);

2. Language variables (Variables for grammar testing: Repetition of sentences-scaled result, Repetition of sentences-descriptive term, Morphological complementation-scaled result, Morphological complementation-descriptive term; Variable for testing the ability to use complex sentences).

\section{Method of conducting research and measuring in- struments}

The development of grammar was examined by means of sub-tests: Repetition of sentences and Mor- phological complementation taken from the Test of Language Development (TOLDP4) (Newcomer \& Hammill, 2008) (adjusted for the Bosnian language). Grammatical sub-test Repetition of sentences, out of a total of 36 tasks, measured the child's ability to repeat sentences. The respondents were expected to repeat the sentence for the examiner in the same manner as the examiner has pronounced. On this variable, the respondent could achieve a maximum of 36 points. A grammatical sub-test The Morphological complementation, with a total of 38 assignments, assessed the child's ability to recognize, understand and use common morphological forms. The morphological complementation was examined so that the examiner read unfinished sentences, and the respondent should supplement the omitted morphological form in the sentence, and the range of results on this variable ranged from 0 to 38 points. Each task could be repeated only once. The test was canceled after 5 consecutive errors. The ability to use complex sentences was assessed in a way to analyze whether the respondent correctly used a complex sentence during the conduct of the test. To test the ability to use complex sentences in children, a "Handbook for Reynell Development Speech Charts" was used (Reynell \& Huntley, 1985) (adjusted for Croatian language, Lovrić, 1995). This task was positively evaluated if the respondent correctly used a complex sentence during the conduct of the test. A complex sentence is defined as the sentence consisting of two or more simple sentences that constitute a unique whole in the sense, structural and intonation aspect. A complex sentence is a linguistic expression of a unique complex thought (Minović \& Ajanović, 1989; Jahić et al., 2000). The correct complex sentence has one main, independent sentence and a subordinate or dependent sentence or sentences (Reynell \& Huntley, 1985).

\section{Statistical data processing}

In statistical data processing for each variable, the basic statistical parameters are calculated: arithmetic mean, standard deviation, minimum and maximum result, frequencies. To test the differences between stuttering children and non-stuttering children, the t-test for an independent sample at a level of significance of 5\% was used. Distribution variables are shown tabular and graphical. The data was processed using the statistical software SPSS 20.0 for Windows. 


\section{RESULTS}

The development of grammar in stuttering children and non-stuttering children was questioned by subtests Repetition of sentences and Morphological complementation. The results of descriptive statistics have shown that the average value of the variable Repetition of sentences scaled results in the stuttering group was 13.06 points, with a range of results ranging from 1 to 17 points. In children who do not stutter, for the average result of the variable Repetition of the sentence, the scaled score was 15.06 points, and the results ranged from 12 to 17 points (Table 1). When we take into account the descriptive terms, we can conclude that the ability to repeat sentences in stuttering children was above the average level, and in children who do not stutter at an excellent level.

Table 1. Descriptive statistics of the variable Repetition of sentences in stuttering children and non-stuttering children

\begin{tabular}{lccccc}
\hline Respondents & $\mathbf{N}$ & $\overline{\mathbf{x}}$ & SD & Min & Max \\
\hline Stuttering children & 32 & 13.06 & 3.172 & 1 & 17 \\
Non-stuttering children & 32 & 15.06 & 1.318 & 12 & 17 \\
\hline
\end{tabular}

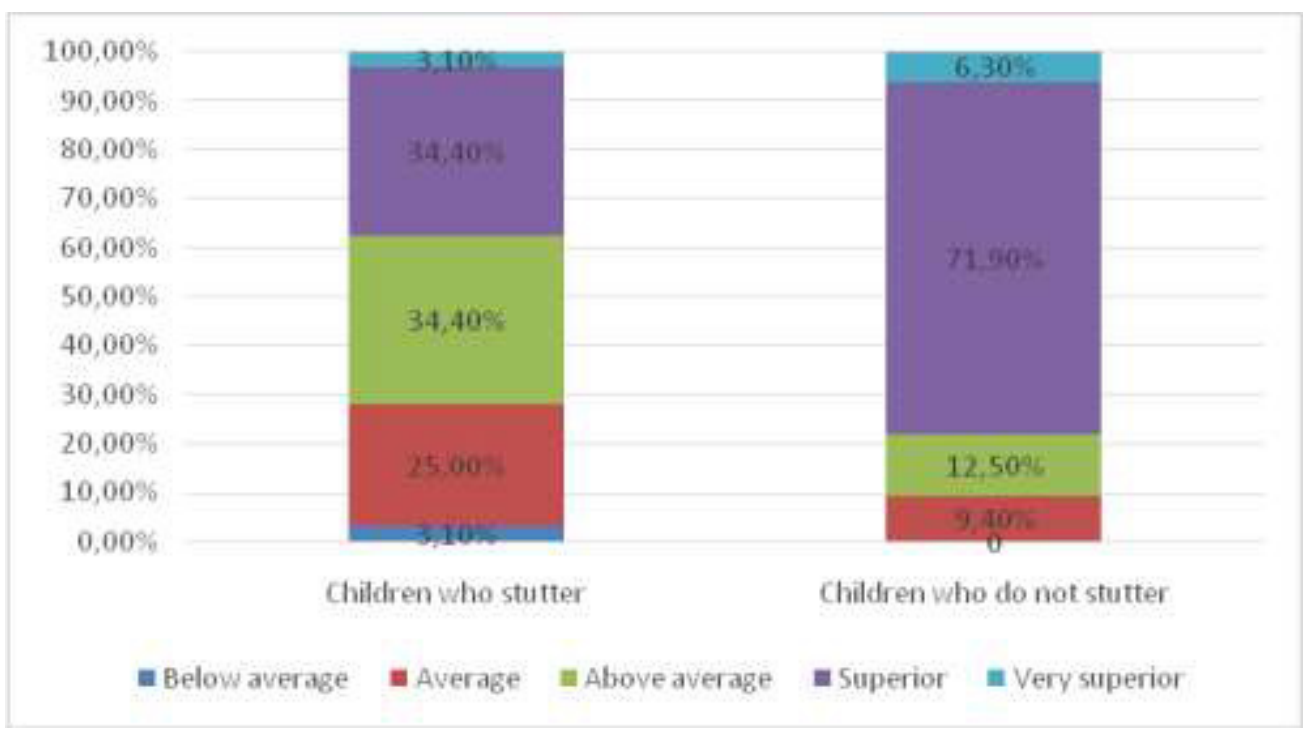

Figure 1. Graphic representation of descriptive grades achieved on the variable Repetition of sentences in stuttering children and non-stuttering children

Figure 1 shows the descriptive grades that the respondents achieved on the Repetition of sentences variable. In stuttering children one respondent was below the average and another was at the very superior level (3.1\%), eight respondents $(25 \%)$ were on an average level, eleven respondents (34.4\%) were above the average and superior levels. In the group of nonstuttering children, 3 respondents $(9.4 \%)$ showed average knowledge, 4 respondents $(12.5 \%)$ were above the average level, 23 respondents showed superior knowledge $(71.9 \%)$, while two fluent respondents were very superior at Repeating of sentences (6.3\%). Using the t-test, results of differences in the repetition ability of the sentences for the entire sample were obtained. The analysis shows that there is a statistically significant difference between children who stutter and children who do not stutter in the examined ability, where the $p$ value was .002 (Table 2).

Table 2. Determining the difference between stuttering children and non-stuttering children based on the variable Repetition of sentences

\begin{tabular}{|c|c|c|c|c|c|c|c|}
\hline \multirow[t]{2}{*}{ Variable } & \multicolumn{2}{|c|}{ Stuttering children } & \multicolumn{2}{|c|}{$\underline{\text { Non-stuttering children }}$} & \multirow[t]{2}{*}{ df } & \multirow[t]{2}{*}{$t$-test } & \multirow[t]{2}{*}{$p$} \\
\hline & $\overline{\mathbf{x}}_{1}$ & $\mathbf{S D}_{1}$ & $\overline{\mathbf{x}}_{2}$ & $\mathrm{SD}_{2}$ & & & \\
\hline $\begin{array}{l}\text { Repetition of } \\
\text { sentences }\end{array}$ & 13.06 & 3.172 & 15.06 & 1.318 & 62 & -3.294 & $.002 *$ \\
\hline
\end{tabular}


The results of descriptive statistics of stuttering children and non-stuttering children on the variable Morphological complementation-scaled result is shown in Table 3 . The average value of the variable Morphological complementation-scaled result in stuttering children was 13.47 points with a standard deviation of 2.032 points. In non-stuttering respondents, the average value of the variable Morphological complementation-scaled result was 14.59 points, with a standard deviation of 1.72 points. When we take into account the descriptive terms for the assessment of Morphological complementation, which ranges from below the average to the very superior, we can establish that the Morphological complementation in stuttering children corresponds to a grade above the average, and in the group of children who do not stutter, the grade is superior.

Table 3. Descriptive statistics of the variable Morphological complementation in stuttering children and non-stuttering children

\begin{tabular}{lccccc}
\hline Respondents & $\mathbf{N}$ & $\overline{\mathbf{x}}$ & SD & Min & Max \\
\hline Stuttering children & 32 & 13.47 & 2.032 & 6 & 16 \\
Non-stuttering children & 32 & 14.59 & 1.720 & 9 & 18 \\
\hline
\end{tabular}

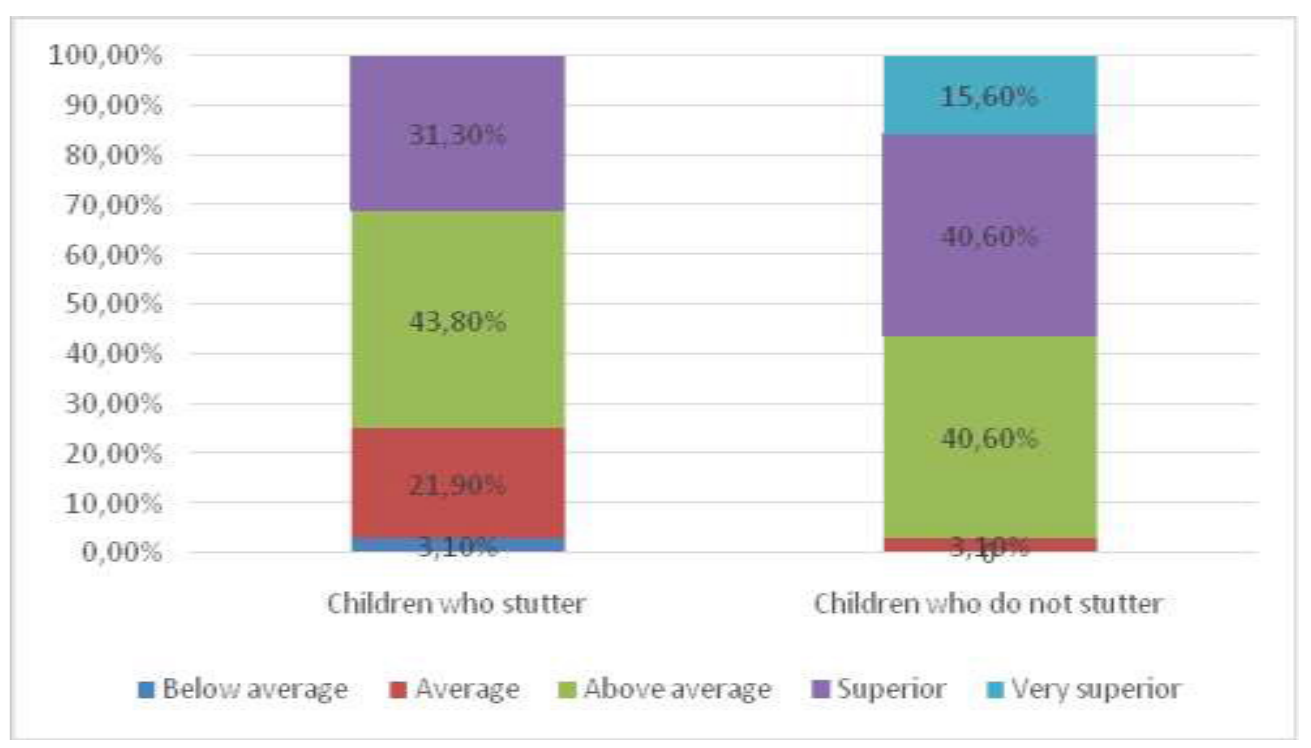

Figure 2. Graphic representation of descriptive grades achieved on the variable Morphological complementation in stuttering children and non-stuttering children

Figure 2 graphically depicts descriptive grades that stuttering children and non-stuttering children achieved on the Morphological complementation variable. In the stuttering children, one respondent $(3.1 \%)$ showed below the average morphological complementation ability, 7 respondents $(21.9 \%)$ achieved an average level, 14 respondents (43.8\%) above the average level, and ten, respectively $31.3 \%$ of respondents showed superior knowledge during morphological complementation. In a group of nonstuttering children, one respondent had an average result of morphological complementing ability (3.1\%), thirteen respondents each (40.6\%) showed above average and superior knowledge, while five respondents or $15.6 \%$ demonstrated very superior knowledge in morphological complementation.

Table 4 shows the results of the obtained statistical levels of significance of the differences for the whole sample compared to the Morphological complementation variable. The results obtained show that there is a statistically significant difference in the morphological complementation capabilities between the experimental and the control group, where the $p$ value was .020. 
Table 4. Determining the differences between stuttering children and non-stuttering children based on the variable Morphological complementation

\begin{tabular}{|c|c|c|c|c|c|c|c|}
\hline \multirow[t]{2}{*}{ Variable } & \multicolumn{2}{|c|}{ Stuttering children } & \multicolumn{2}{|c|}{ Non-stuttering children } & \multirow[t]{2}{*}{ df } & \multirow[t]{2}{*}{$t$-test } & \multirow[t]{2}{*}{$p$} \\
\hline & $\overline{\mathbf{x}}_{1}$ & SD $_{1}$ & $\overline{\mathbf{x}}_{2}$ & $\mathbf{S D}_{2}$ & & & \\
\hline $\begin{array}{l}\text { Morphological } \\
\text { complementation }\end{array}$ & 13.47 & 2.032 & 14.59 & 1.720 & 62 & -2.391 & $.020 *$ \\
\hline
\end{tabular}

To test the use of grammatically complex sentences in stuttering children and non-stuttering children, the "Handbook for Reynell Development Scales" (Reynell \& Huntley, 1985) was used. In order for the task to be graded positively, the respondent should correctly use a complex sentence during the course of the examination. After analyzing the results, it was found that all subjects of the control group used a complex sentence in their spontaneous speech. In the experimental group, only two respondents in their spontaneous speech did not use a complex sentence. After using the t-test for the comparison of stuttering children and non-stuttering children in the use of a complex sentence in spontaneous speech, it was found that there is no statistically significant difference in the use of complex sentences among the mentioned groups of respondents $(\mathrm{p}=.156)(\mathrm{t}$-test 1.438).

\section{DISCUSSION}

Grammatical features begin to be observed when certain grammatical errors appear during the speech or when a child has problems understanding. At that point, a meta-language approach to language processing can be helpful. Automatic morpho-syntax processing is present in all native speakers. It is apparent that speakers do not have awareness of the grammatical characteristics of the language they listen to or produce, but interpreted the message by built-in automatisms (Ljubešić, Blaži, \& Bolfan-Stošić, 1993). Repetition of sentences as a research and / or diagnostic technique is based on observations that/when the child does not repeat speech mechanically. Child repetition of sentences or words is an active process of cognitive processing of a given word/sentence, and the reproduction itself gives us insight into the child's semantic and grammatical language governance (Rood \& Braine, 1979, according to Ljubešić et al., 1993). The ability to repeat sentences in stuttering children in this research was above average and in non-stuttering children, it was at the superior level. Stuttering children received a fairly wide range of results from 1 to 17 points, and the lowest score on the sub-test Repetition of sentences in the control group was 12 points. By examining the differences between stuttering children and non-stuttering children, it was found that there was a statistically significant difference between the analyzed groups on the Repetition of sentences variable. We can conclude that stuttering children have a significantly weaker repetition of sentences compared to non-stuttering children. However, it is important to emphasize that, regardless of the lower results compared to their fluent peers, stuttering children have achieved above the average ability to repeat sentences. Observing the ability of morphological complementation in stuttering children, the results showed above average values, and in the group of children who do not stutter superior values. We can conclude that stuttering children have shown significantly lower results than children who do not stutter on Morphological complementation, but again we emphasize that their abilities to recognize, understand and use common morphological forms have been above average.

As we already pointed out in the introductory part, the results of the study of the linguistic abilities of the stuttering children are not unambiguous, or some studies have shown that the linguistic abilities in stuttering children are weaker than in those who do not stutter, while the results of some other studies have not shown differences in those abilities amongst stuttering children and their fluent speaking peers (Kutnjak, Mance, \& LekoKrhen, 2016). Regardless of the lack of uniformity of the results, the results obtained in this study agree with the majority of research by other authors who claim that stuttering children have less developed grammatical functions than children with fluent speech. Thus, Salihović (2005) states that numerous studies have shown that stuttering children differ significantly from children who do not stutter on variables such as the level of receptive vocabulary, the average length of the expression, and expressive and receptive syntax. Anderson and Conture (2004) examined the ability of syntactic processing between stuttering children and non-stuttering children 3.3 to 5.5 years old. 
The results indicate that stuttering children may have difficulty in quickly, effectively planning and / or downloading units of the sentence structure, as well as in difficulties that may contribute to their inability to tighten the fluency of speech-language production. Also, Anderson and Conture (2000) and Pellowski and Conture (2005) found that stuttering children have poorer linguistic abilities than non-stuttering children. Bajaj et al. (2004), during the examination of grammar in stuttering children and non-stuttering children, have achieved results that suggest that children who do not stutter outdone children who stutter in the sense of assessing semantic and syntactic deformed sentences. Non-stuttering children showed statistically better results on these tasks than stuttering children. Unlike them, Watkins, Yairi and Ambrose (1999) found more developed language skills in stuttering children compared to their non-stuttering peers. Research on children who began to stutter by age four showed better linguistic and non-verbal cognitive abilities and better quality of life compared to children who did not stutter at the age of 4 (Reilly et al., 2013) . However, Nippold and Schwarz (1991) found that there was no difference between these two groups. It is not yet known whether stuttering occurs as a consequence of the difficulties between semantic and morphological encryption, certain slowdowns when returning to complex phonological speech patterns or other disturbances. Lately, more questions are being considered about whether stuttering is associated with linguistic processes above motoric planning. More and more, it is concluded that stuttering is partially caused by linguistic processes (Anderson \& Conture, 2000).

After comparing stuttering children and children who do not stutter in the use of complex sentences in a spontaneous speech in this study, it has been established that there is no statistically significant difference in the use of complex sentences between experimental and control groups. Both stuttering children and non-stuttering children used complex sentences in their spontaneous speech. As we have explained in the previous section of the discussion, there is also no consensus on this subject, that is, the results of the research of other authors are different, with some research being confirmed, and they negate the existence of a difference in the use of complex sentences in the speech of children who stutter and children who do not stutter. The authors find it possible that stuttering children avoid using compound sentences in their speech because the fact is that more stuttering non-fluency will occur in more complex sentences. There is still no answer as to whether this increase in the number of non-fluency is affected by linguistic complexity or an increase in fear during the pronunciation of more complex statements. Owens (2005) points out that the sentence and its meaning are more important than the meaning of individual words, that is, sentences represent a greater meaning than the sum of individual words. The sentence is not just a set of words; it is also a link between these words. Ward (2006) states that stuttering tends to occur where linguistic concerns are high, that is, by increasing the appearance of more complex linguistic structures. As the preschool age coincides with the rise of linguistic expression, at the same time, there is an accelerated motor development of speech. It is known that stuttering is associated with reduced speech motoric skills, and it is also the case that the moments of linguistic complexity tend to bring it in close with the complexity of speech motoric. Thus, Wall (1980, according to Howell \& Au-Yeung, 1995) compared a group of stuttering children aged 5 and 6 and a group of their peers with fluent speech. The author has reported that fluent speakers use a number of complex sentences rather than stuttering children. In other words, stuttering children use simpler syntax and incomplete clauses. Kadi-Hanifi and Howell (1992, according to Howell \& Au-Yeung, 1995) investigated the frequency of the use of various types of sentences among stuttering children and non-stuttering children aged 4,5 , and 11 . The authors found that among the stuttering children, compared to a balanced age group of fluent speakers, there was no difference in the frequency of using different sentence categories. Weber-Fox and Hampton (2008) indicate that stuttering is associated with differences in the activation of language processing, even in the absence of open planning and speech production. Their results are consistent with the multi-factorial model of stuttering (Smith, 1990; Smith \& Kelly, 1997, according to Weber-Fox \& Hampton, 2008) and show that the disorder associated with differences in brain morphology affects not only the coordination of the speech system engine, but it can also reflect the effectiveness of operations related to the integration and differentiation of semantic and syntax flows involved in language processing.

From a clinical perspective, if children have higher speech velocity, longer statements or greater complexity at the end of a long speech pattern, interventions that reduce velocity, length of statements, and complexity can help children in the acquisition of speech fluidity. 
A closer examination of the parameters of the length of the statement, the grammatical complexity and the velocity of speech in each child would provide additional information to clinicians and researchers on factors affecting disfluency in pre-school children (Sawyer, Chon \& Abrose, 2008). Stewart and Turnbull (1997) explain that if poor language skills reduce child's capacity for fluent speech, then working on language skills should serve to increase the capacity of the language. During this time, the child's speech fluency must be carefully taken into account in order to see if there are negative consequences, because if certain consequences occur, it is necessary to revise the work program. On the other hand, ignoring language difficulties limits the child's potential to develop effective communication. Begić and Babić (2017) also emphasize that during the assessment process and the diagnosis of stuttering children, the ability of the child to use complex linguistic statements should be assessed, and the frequency of dysfunction should be assessed in relation to the complexity of the statements. Precise diagnostics would provide guidelines for the treatment of stuttering in terms of implementation approaches and strategies that involve a gradual increase in the length and complexity of the statements of children who stutter during speech and linguistic therapy. During the process of evaluation and diagnosis of children who are stuttering, special attention should be paid to their linguistic abilities (Begić, Mrkonjić, \& Salihović, 2014). The results of the research are also in line with the research results of Kefalianos, Onslow, Packman, Vogel and Pezic (2017), which also suggest that there can be associations between the development of language abilities and the recovery of stuttering. Future research should be directed to clearly identify the guideline of this link.

Research shows that stuttering people improve speech fluency at the heart of language, or by limiting the use of grammar. Namely, the stutterers use less sentences statements, which are simpler in structure, and use less models for expressing opinions and attitudes, which potentially less initiate communication and less enter into communication interaction (Spencer et al., 2009, according to Onslow, 2018). Considering that in our research, the respondents are quite young, that is, preschool children and first-class children, assuming that they have not yet developed a consciousness of speech difficulties, nor have fears developed about speech and speech situations, and have not developed negative attitudes towards communication, and thus children did not avoid using spontaneous speech, in addition to simple and complex sentences, which can often be the case with adolescents and adults who stutter.

\section{CONCLUSION}

The results show that stuttering children and nonstuttering children do not have the same level of grammar development. Stuttering children exhibit lower abilities during repetition of sentences, as well as recognition, understanding and use of common morphological forms. However, we emphasize that children who stutter, regardless of the significantly lower results compared to their fluent speaking peers, have shown above-average grammatical abilities. Also, it is important to point out that in children who are stuttering the results showed a much wider range of results obtained and standard deviation. The results also showed that children who stutter and children who do not stutter in their spontaneous speech use complex sentences. Therefore, the causal link between language skills/disorders and stuttering has not been established so far, but the issue to be considered is the relationship between language skills and speech itself, and whether stuttering is the cause or consequence of poorer results in the area of language skills. Precise data on this issue, especially the results of research conducted in the official languages of Bosnia and Herzegovina precisely because of the lack of data from our speaking area, can greatly assist in the detection of factors related to stuttering, linguistic characteristics of stuttering children, and treatment plans that will certainly include grammati$\mathrm{cal} /$ the language component of such children. The results also point to the conclusion that the diagnostic and rehabilitation processes of stuttering children should necessarily include assessment of language abilities, and determine whether stuttering can lead to linguistic difficulties or, on the other hand, whether language difficulties can lead to the occurrence of non-fluency in the child's speech. Language models for speech therapists should give guidelines on which specific aspects of language should be evaluated and later treated. If the child shows both linguistic disorder and stuttering, the treatment plan should include the treatment of both disorders at the same time, combining methods of language stimulation and increasing speech fluency. Namely, understanding the child's grammatical development/disorder and its impact on stuttering, or how the linguistic aspects of speech-language planning and production can contribute to, or aggravate the development of stuttering in children, should greatly help us in the planning of treatment, and it is certainly suggested that further research should be carried out on the mentioned topic. 


\section{REFERENCES}

Anderson, J.D., \& Conture, E.G. (2000). Language abilities of children who stutter: A preliminary study. Journal of Fluency Disorders, 25, 283-304. https://doi.org/10.1016/S0094730X(00)00089-9.

Anderson, J.D., \& Conture, E.G. (2004). Sentence-structure priming in young children who do and do not stutter. Journal of Speech Language and Hearing Research, 47(3), 552-71. doi: 10.1044/1092-4388(2004/043).

Bajaj, A., Hodson, B., \& Schommer-Aikins, M. (2004). Performance on phonological and grammatical awareness metalinguistic tasks by children who stutter and their fluent peers. Journal of Fluency Disorders, 29(1), 63-77. doi: 10.1016/j. jfludis.2004.01.001.

Begić, L., \& Babić, B. (2017). Influence of length of sentences on the frequency of speech disfluencies in children who stutter. $\mathrm{Hu}$ man Research in Rehabilitation, 7(1), 50-55. doi: 10.21554/ hrr.041706.

Begić, L., Mrkonjić, Z., \& Salihović, N. (2014). Development of phonological awareness of stuttering children and children with fluent speech. Journal of special education and rehabilitation, 15(12), 59-74. doi: 10.2478/JSER-2014-0004.

Duranović, M., Begić, L., Jovanović-Simić, N., \& Rahmanović, D. (2018). Komunikacija i mucanje. Foča. Univerzitet u Istočnom Sarajevu, Medicinski fakultet Foča.

Finneran, D.A., Leonard, L.B., \& Miller, C.A. (2009). Speech Disruptions in the Sentence Formulation of School-Age Children with Specific Language Impairment. Int Journal of Language Communication Disorders, 44(3), 271-286. doi: 10.1080/13682820902841385.

Howell, P., \& Au-Yeung, J. (1995). Syntactic determinants of stuttering in the spontaneous speech of normally fluent and stuttering children. Journal of Fluency Disorders, 20, 317-330. https://doi.org/10.1016/0094-730X(94)00012-I.

Jahić, DŽ.,Halilović, S., \& Palić, I. (2000). Gramatika bosanskog jezika. Zenica: Dom štampe.

Kefalianos, E., Onslow, M., Packman, A., Vogel, A., \& Pezic, A. (2017). The History of Stuttering by 7 Years of Age: FollowUp of a Prospective Community Cohort. Journal of Speech Language and Hearing Research, 60(10), 2828-2839. doi: 10.1044/2017 JSLHR-S-16-0205.

Khan, N. (2015). The Effect of Stuttering on Speech andLearning process A Case Study. International Journal on Studies in English Language and Literature (IJSELL), 3(4), 89-103. https://www.arcjournals.org/pdfs/ijsell/v3-i4/13.pdf.

Kutnjak, N., Mance, L., \& LekoKrhen, A. (2016). Jezične sposobnosti djece koja mucaju. Logopedija, 6(2), 69-74. https://doi. org/10.31299/log.6.2.4.

Ljubešić, M., Blaži, D., \& Bolfan-Stošić, N. (1993). Posebne jezičke teškoće djece osnovnoškolske dobi. Govor, 10(1), 17-37. https://hrcak.srce.hr/17623.

Newcomer, P.L., \& Hammill, D.D. (2008). Test of language development. Austin, Texas. Pro-ed.

Nippold, M. (2004). The Child Stutters and Has a Phonological Disorder: How Should Treatment Proceed? In: Bothe, A.K. (2004). (Ed). Evidence-Based Treatment of Stuttering Empirical Bases and Clinical Applications. Mahwah, New Jersey. Lawrence Erlbaum Associates, Inc., 97-116. https:// the-eye.eu/public/Books/BioMed/Evidence $\% 20$ Based $\% 20$ Treatment $\% 20$ of $\% 20$ Stuttering $\% 20-\% 20$ A.\%20Bothe $\% 20$ $\% 28$ ed $\% 29 \% 20 \% 282004 \% 29 \% 20 W W . p d f$.
Nippold, M.A., \& Schwarz, I.E. (1991). Narrative ability in schoolage stuttering boys: A preliminary investigation. Journal of Fluency Disorders, 16(5-6), 289-308. https://doi. org/10.1016/0094-730X(91)90041-A.

Onslow, M. (2018). Stuttering and Its Treatment-Eleven lectures. Sydney. Australian Stuttering Research Centre, The University of Sydney. https://www.uts.edu.au/sites/default/ files/201810/Stuttering\%20and $\% 20$ its $\% 20$ Treatment $\% 20$ -\%20Eleven\%20Lectures\%20April\%202018_0.pdf.

Owens, R.E. Jr. (2005). Language development: An Introduction, 6th ed. New York. Pearson Education, Inc.

Pellowski, M.W., \& Conture, E. (2005). Lexical priming in picture naming of young children who do and do not stutter. Journal of Speech, Language, and Hearing Research, 48(2), 278-294. doi: 10.1044/1092-4388(2005/019).

Ratner, N.B. (1995). Treating the child who stutter with concomitant language or phonological impairment. Language, speech and hearing services in the school, 26, 180-186.

Reilly, S., Onslow, M., Packman, A., Cini, E., Conway, L., Ukoumunne, O., \& Wake, M. (2013). Natural history of stuttering up to 4 years of age: A prospective communitybased study. Pediatrics, 132(3), 459-467. doi: 10.1542/ peds.2012-3067.

Reynell, J.K., \& Huntley, M. (1985). Priručnik za Reynell razvojne ljestvice govora, Drugo revidirano izdanje. Jastrebarsko: Naklada Slap.

Salihović, N. (2005). Poremećaji tečnosti govora. Tuzla: Univerzitet u Tuzli.

Sawyer, J., Chon, H., \& Ambrose, N.G. (2008). Influences of rate, leght and complexity on speech disfluency in a single-speech sample in preschool children who stutter. Journal of Fluency Disorders, 33(3), 220-40. doi: 10.1016/j.jfludis.2008.06.003.

Stewart, T., \& Turnbull, J. (1997). Working with Dysfluent Children. Brackley. Speech mark publishing Ltd.

Ward, D. (2006). Stuttering and Cluttering. Frameworks for understanding and treatment. Hove and New York. Taylor \& Francis e-Library.

Watkins, R.V., Yairi, E., \& Ambrose, N.G. (1999). Early childhood stuttering III: Initial status of expresive language abilities. Jounal of Speech, Language and Hearing Research 42(5), 1125-1135. doi: 10.1044/jslhr.4205.1125.

Watkins, R.V., \& Johnson, B.W. (2004). Language Abilities in Children Who Stutter: Toward Improved Research and Clinical Applications. Language, Speech, And Hearing Servicesin Schools, 35(1), 82-89.

Weber-Fox, C., \& Hampton, A. (2008). Stuttering and Natural Speech Processing of Semantic and Syntactic Constraints on Verbs. Journal of Speech, Language Hearing Research 51(5), 1058-1071. doi: 10.1044/1092-4388(2008/07-0164). 